Nouvelles perspectives en sciences sociales

Revue internationale de systémique complexe et d'études relationnelles

\title{
Inégalités et minorisation des identités chez les femmes artistes dans la francophonie canadienne
}

\section{Anne Robineau}

Volume 8, numéro 2, mai 2013

Sur le thème de représentations identitaires et expressions culturelles de la francophonie canadienne à travers ses pratiques artistiques et médiatiques

URI : https://id.erudit.org/iderudit/1016474ar

DOI : https://doi.org/10.7202/1016474ar

Aller au sommaire du numéro

Éditeur(s)

Prise de parole

ISSN

1712-8307 (imprimé)

1918-7475 (numérique)

Découvrir la revue

Citer cet article

Robineau, A. (2013). Inégalités et minorisation des identités chez les femmes artistes dans la francophonie canadienne. Nouvelles perspectives en sciences sociales, 8(2), 145-174. https://doi.org/10.7202/1016474ar

\section{Résumé de l'article}

Bien que les femmes artistes souhaitent une reconnaissance de leurs oeuvres indépendamment de leur genre, de leur langue ou de l'appartenance à un groupe culturel, leurs conditions de pratique sont parfois liées à ces statuts. À travers une étude sociodémographique des artistes de la francophonie canadienne, nous analysons les données relatives aux femmes artistes. Nous montrons que, bien qu'elles détiennent des diplômes plus élevés que leurs homologues masculins, elles sont considérablement moins bien rémunérées que ces derniers. Nous cherchons alors à comprendre si des processus de minorisation des femmes de l'activité professionnelle artistique en milieu francophone peuvent expliquer certaines inégalités et comment la production des discours sur l'identité (femme, francophonie, minorité linguistique) renforce la tension entre l'art et la perspective identitaire. 


\title{
Inégalités et minorisation des identités chez les femmes artistes dans la francophonie canadienne
}

\author{
Anne Robineau \\ Institut canadien de recherche \\ sur les minorités linguistiques (ICRML), Moncton
}

\section{Introduction}

$\mathbf{L}$

es statistiques exposent régulièrement les inégalités qui persistent encore entre les hommes et les femmes sur le marché du travail ${ }^{1}$. De nombreuses recherches mettent alors en cause des représentations traditionnelles tenaces sur le rôle des femmes dans la société ${ }^{2}$. D’une part, ces représentations ainsi qu'un

$1 \quad$ Statistique Canada, Femmes au Canada : rapport statistique fondé sur le sexe, 2010-2011, sixième édition, 89-503-XIF, Ottawa, 2011; "La première édition de Femmes au Canada a débuté en 1985, soit la même année que la troisième Conférence mondiale sur les femmes organisée par les Nations Unies à Nairobi. Le rapport découlant de cette conférence révèle que [traduction] "le manque de données fiables sur les femmes nuit à l'évaluation de leur progrès dans divers domaines et qu'il faudrait s'investir davantage afin d'obtenir des données sur l'évolution des sexes", dans NATIONS UNIES, Rapport de la Conférence mondiale chargée d'examiner et d'évaluer les résultats de la Décennie des Nations Unies pour la femme : égalité, développement et paix, New York, Nations Unies. ", 1986, [En ligne] http://www.statcan.gc.ca/ pub/89-503-x/89-503-x2010001-fra.htm, consulté le 15 janvier 2013.

2 Francine Descarries, "Le Mouvement des femmes et ses courants de pensée: essai de typologie ", Les Documents de l'ICREF, n ${ }^{\circ} 19$, Ottawa, Institut canadien de recherches sur les femmes, 1998; Francine Descarries, Chronologie 
certain nombre d'obstacles propres au marché du travail entravent l'accès des femmes à l'emploi. D'autre part, quand les femmes obtiennent un emploi, ces représentations et ces obstacles diminuent leur chance de poursuivre une carrière professionnelle équivalente à celle des hommes.

Selon certaines auteures ${ }^{3}$, les mondes artistiques n'échapperaient pas à cette dynamique qui tend à minoriser la place des femmes dans l'activité socioéconomique. Cette minorisation peut se traduire par des revenus moindres pour une tâche similaire, des difficultés à intégrer les sphères du pouvoir et des lieux de décision concernant leurs pratiques telles des comités de pairs, des conseils d'administration, des réseaux de diffusion, etc. Cela a un impact sur la reconnaissance de leurs œuvres, la place qu'elles occupent dans l'histoire de leur discipline artistique et l'influence qu'elles peuvent elles-mêmes avoir dans leur sphère d'activitét . De plus, dans le cadre de celles qui exercent leur profession au sein de communautés francophones en milieu minoritaire, il y a lieu de se demander si un autre processus de minorisation, celui-ci lié à la langue, n'a pas un effet cumulatif sur les inégalités dont les femmes font l'objet ou pas. En effet, à l'extérieur du Québec, les artistes disposent de ressources plus

de l'histoire des femmes au Québec et rappel d'événements marquants à travers le monde, 2007, [en ligne] http://www.unites.uqam.ca/arir/pdf/chronologieNouvelleVersionJuin2007.pdf., consulté le 15 janvier 2013; Danièle Kergoat, "Division sexuelle du travail et rapports sociaux de sexe ", dans Helena Hirata, Françoise Laborie, Hélène Le Doaré et Danièle Senotier (dir.), Dictionnaire critique du féminisme, Paris, Presses universitaires de France, 2000, p. 35-44; Danièle Kergoat, "Rapports sociaux et division du travail entre les sexes ", dans Margaret Maruani (dir.), Femmes, genre et sociétés, Paris, La Découverte, 2005, p. 94-101.

3 Agnese Fidecaro et Stéphanie Lachat (dir.), Profession: créatrice. La place des femmes dans le champ artistique, Actes du colloque de l'Université de Genève (18 et 19 juin 2004), Lausanne, Antipodes, 2007; Rose-Marie Arbour, " Le Cercle des automatistes et la différence des femmes», Études françaises, vol. 34, $\mathrm{n}^{\text {os }}$ 2-3, 1998, p. 157-173; Marie-Thérèse Lefebvre, La Création musicale des femmes au Québec, Montréal, Les Éditions du remue-ménage, 1991; Anna Lupien, De la cuisine au studio, Montréal, Les Éditions du remue-ménage, 2012.

4 Anne Robineau, "Musique, femme et création musicale ", dans Dire, Université de Montréal, vol. 12, n 1, janvier 2004, p. 19-22. 
rares en français pendant les différentes étapes de leur carrière (formation, création, diffusion), ce qui peut les isoler plus facilement.

Dans cet article, après avoir exposé, dans un premier temps, des données statistiques sur les artistes de la francophonie canadienne, nous nous proposons d'examiner, dans un deuxième temps, les hypothèses qui pourraient expliquer les processus de minorisation à travers la professionnalisation différenciée entre les hommes et les femmes artistes. Dans un troisième temps, nous aborderons les conditions de pratiques des artistes en milieu minoritaire francophone afin de saisir dans quelle mesure certains facteurs pourraient faire obstacle à leur carrière professionnelle.

\section{Portrait sociodémographique et professionnalisation des femmes artistes}

Notre questionnement quant aux inégalités entre les hommes et les femmes artistes provient d'une étude récente menée sur les artistes qui exercent leur profession au sein des communautés francophones en situation minoritaire 5 . Dans cette étude, nous avons, entre autres, dressé un portrait sociodémographique des artistes de la francophonie canadienne dans lequel des différences très importantes de revenu entre les hommes et les femmes sont apparues. En nous appuyant sur les données du recensement ${ }^{6}$, nous avons également mis de l'avant plusieurs caractéristiques sociodémographiques des femmes artistes dont nous nous proposons de donner tout d'abord un bref aperçu.

Nous comparons, entre autres, la situation des artistes francocanadiennes avec celle de l'ensemble des autres artistes au Canada, mais aussi avec certaines caractéristiques de l'ensemble de la population active canadienne. En somme, nous cherchons non seulement à savoir plus précisément si les femmes artistes sont aussi bien rémunérées pour leur travail par rapport à leurs

$5 \quad$ Anne Robineau, Un Regard actuel sur la situation des artistes dans la francophonie canadienne, Rapport de recherche pour le Conseil des arts du Canada, Moncton, Institut canadien de recherche sur les minorités linguistiques (2013, à paraître).

6 Statistique Canada, 2006. 
homologues masculins et par rapport au reste de la population active, mais également s'il existe des différences entre les femmes et les hommes artistes quant à leur représentativité en fonction des disciplines artistiques ou de leur niveau de scolarité. Nous tenons aussi à comparer ces caractéristiques en fonction du contexte francophone au Canada qui peut faire varier considérablement les chiffres lorsqu'on inclut ou exclut le Québec. Avant de décrire ces données, nous aimerions rappeler sur quoi se basait le choix des variables statistiques utilisées afin de bien saisir les limites méthodologiques de l'étude susmentionnée?

Choix des variables et limites méthodologiques

Premièrement, l'étude en question devait tenir compte de la situation des artistes issus des communautés francophones en situation minoritaire en fonction de six disciplines artistiques : les arts médiatiques, les arts visuels, la musique, la danse, le théâtre et les lettres. Il fallait donc trouver des variables qui correspondraient à ces disciplines artistiques. Pour cela, nous avons choisi d'utiliser les données recueillies par Statistique Canada selon le classement national des professions. Il faut noter qu'une discipline peut recouvrir plusieurs professions comme cela s'est avéré pour la musique. Ainsi, sept catégories socioprofessionnelles de ce classement ${ }^{8}$ ont servi à obtenir des données sur les professions artistiques dans la francophonie canadienne.

Ce choix de variable constitue une première limite étant donné que seuls les artistes qui ont 15 ans et plus et qui déclarent un revenu sont comptabilisés. Autrement dit, les chiffres sont plus conservateurs selon ces critères que d'après d'autres définitions

Anne Robineau, Un Regard actuel sur la situation des artistes dans la francophonie canadienne, op. cit.

8 Arts médiatiques (F031: Producteurs / productrices, réalisateurs / réalisatrices, chorégraphes et personnel assimilé); Arts visuels (F036 : Peintres, sculpteurs / sculpteures et autres artistes des arts visuels); Musique et chanson (F032: Chefs d'orchestre, compositeurs / compositrices et arrangeurs / arrangeuses) et (F033 : Musiciens / musiciennes et chanteurs / chanteuses); Danse (F034 : Danseurs / danseuses); Lettres et édition (F021 : Auteurs / auteures, rédacteurs / rédactrices et écrivains / écrivaines); Théâtre (F035 : Acteurs / actrices et comédiens / comédiennes). 
du statut d'artiste. Par exemple, un écrivain qui s'identifierait à son statut d'artiste, mais qui tirerait son revenu d'une autre profession, ne figurerait pas dans ce classement, et ce, indépendamment de la notoriété qu'il pourrait avoir comme auteur ou de sa vocation d'artiste. Qui plus est, chaque catégorie socioprofessionnelle regroupe sans distinction des artistes dont le langage artistique et les codes esthétiques peuvent être très différents. Cela se répercute sur leurs réseaux de diffusion et leurs stratégies auprès du public. En effet, certains artistes classés dans la même catégorie vont intégrer les réseaux plus commerciaux des industries culturelles tandis que d'autres vont plutôt choisir la voie de la création indépendante et de la production autogérée. Cela a un impact inévitable sur leur revenu respectif sans qu'on puisse le discerner dans les statistiques. Force est d'admettre que ces classifications comportent plusieurs lacunes et qu'elles répondent avant tout à des besoins administratifs des institutions gouvernementales et des organismes subventionnaires. Cependant, malgré ces lacunes, le choix de ces variables offre deux avantages: il permet d'avoir une dimension comparative entre, d'une part, les différentes disciplines artistiques étudiées, et, d'autre part, entre l'activité socioprofessionnelle des artistes et celle de l'ensemble de la population active.

Deuxièmement, comme l'étude portait sur la francophonie canadienne, un autre choix de variable a dû être effectué concernant la langue. Comme pour la définition d'un artiste, celle d'un francophone recouvre des dimensions plus larges que ce qui peut être circonscrit par des variables linguistiques. Dans le cadre cette étude, nous avions choisi la variable de la première langue officielle parlée (PLOP) (français; français et anglais) car elle englobe, entre autres, les Canadiens de langue maternelle française et les immigrants, de plus en plus nombreux, qui connaissent et parlent le français ${ }^{9}$. La PLOP est en effet une variable dérivée de trois

$9 \quad$ Nous avions mentionné que « la variable de la langue maternelle (français; français et anglais) serait aussi pertinente, surtout dans les régions où l'immigration est faible. La PLOP a l'avantage d'être plus inclusive, même si une petite partie des francophones peuvent échapper au calcul (ceux qui ont à la fois l'anglais et le français comme langues maternelles et qui parlent l'anglais 
autres variables : la connaissance des deux langues officielles, de la langue maternelle et de la langue parlée le plus souvent à la maison ${ }^{10}$. Les données ont ensuite été réparties en fonction du sexe et de la population totale ${ }^{11}$. Ainsi, pour chaque variable (nombre, revenu, scolarité, etc.), un tableau rend compte de façon détaillée des données relatives aux hommes et aux femmes (voir tableaux en annexe) ${ }^{12}$.

$\mathrm{Si}$ on s'intéresse maintenant aux résultats de cette étude, on constate plusieurs tendances qui caractérisent les femmes artistes. Il faut, néanmoins, interpréter ces résultats avec prudence en raison des limites évoquées plus haut.

le plus souvent à la maison). Cela a une conséquence sur le calcul d'enfants des ayants droits dans le cas de couples exogames. Par contre, comme ce projet de recherche examine plutôt la situation des artistes professionnels et non pas une situation relative au système d'éducation et au nombre d'élèves pouvant fréquenter une école francophone, cette conséquence peut être "minimisée" au profit de celle qui consisterait à ne pas prendre en compte les nouveaux arrivants dont le français est la langue maternelle ou la langue la plus souvent parlée à la maison " (Anne Robineau, Un Regard actuel sur la situation des artistes dans la francophonie canadienne, op. cit.).

10 Pour une définition exacte de la première langue officielle parlée et de la façon dont elle est calculée, se référer au site web de Statistique Canada sur les définitions, les sources de données et méthodes : http://www.statcan.gc.ca/ concepts/definitions/language-langue05-fra.htm

11 Pour mieux comprendre le poids relatif des artistes francophones en situation minoritaire au sein des autres communautés linguistiques d'artistes au Canada, nous avons distingué trois catégories : 1) l'ensemble des artistes canadiens (anglais et français); 2) l'ensemble des artistes francophones de toutes les provinces et des territoires (incluant ceux du Québec); 3) l'ensemble des artistes de la francophonie canadienne en situation minoritaire (excluant ceux du Québec). Ces trois catégories de la population artistique sont mises en relation avec trois autres catégories de la population canadienne : 1) l'ensemble de la population active canadienne; 2) l'ensemble de la population francophone active de toutes les provinces et des territoires (incluant ceux du Québec); 3) l'ensemble de la population francophone active en situation minoritaire (excluant ceux du Québec).

12 L'ensemble des tableaux figure dans le rapport Robineau 2013, Anne Robineau, Un Regard actuel sur la situation des artistes dans la francophonie canadienne, op. cit. 
Des femmes plus nombreuses à exercer une profession artistique Tout d'abord, rappelons que, d'après les données de cette étude ${ }^{13}$, les artistes francophones (sans le Québec) constituent 2,6 \% de l'ensemble des artistes au Canada. En tenant compte des artistes francophones du Québec, cette proportion atteint 19,9\% ( $21,3 \%$ pour les hommes et $18,5 \%$ pour les femmes). Ainsi, près d'un artiste sur cinq au Canada est issu d'un contexte francophone. Que ce soit au Canada ou dans la francophonie canadienne (sans le Québec), les femmes sont plus nombreuses à déclarer une profession artistique : elles seraient $61060 \mathrm{au}$ Canada, 11310 dans la francophonie canadienne et québécoise et 1715 dans la francophonie canadienne en excluant le Québec ${ }^{14}$. Les artistes femmes franco-canadiennes (sans le Québec) représentent alors $0,58 \%$ de la population active des femmes de la francophonie canadienne (sans le Québec) ${ }^{15}$.

Les femmes surreprésentées en danse

La danse est la discipline artistique où les femmes sont le plus représentées, quel que soit leur groupe linguistique. En milieu minoritaire francophone, elles forment $78 \%$ des effectifs de cette discipline et autour de $50 \%$ des effectifs dans toutes les autres disciplines artistiques, sauf en musique, dans la catégorie socioprofessionnelle très spécifique des cheffes d'orchestre et des compositrices $(28,6 \%)^{16}$. Quelques nuances sont également à apporter dès que l'on compare les données sur les femmes artistes franco-canadiennes (sans le Québec) à celles des autres femmes artistes. En effet, les femmes artistes franco-canadiennes (sans le Québec) sont plus nombreuses que leurs consœurs à exercer une profession artistique dans les lettres et l'édition $(58,8 \%)$, en théâtre $(54,0 \%)$ et en arts médiatiques $(49,6 \%)^{17}$.

\footnotetext{
13 Ibid.

14 Tableau 1 en annexe.

15 Tableau 2, en annexe.

16 Tableau 7, en annexe.

17 Tableau 7 , en annexe.
} 
Les femmes artistes plus hautement diplômées

Comme nous l'avons plusieurs fois mentionné, les femmes artistes sont proportionnellement plus nombreuses à détenir les diplômes les plus hauts tout en faisant également partie des catégories les moins rémunérées. Par exemple, les femmes artistes francophones (sans le Québec) sont 47,7 \% à détenir un certificat, un diplôme ou un grade universitaire (équivalent au baccalauréat ou plus) alors que leurs homologues masculins sont $41,3 \%$ à posséder l'un de ces grades ou diplômes ${ }^{18}$. Ce pourcentage est de $45,3 \%$ pour les femmes artistes francophones incluant celles du Québec (37,0 \% pour les hommes) et de $47,0 \%$ pour l'ensemble des femmes artistes au Canada (38,6\% pour les hommes). Elles sont également peu nombreuses à ne détenir aucun diplôme (6,4\%) contrairement à l'ensemble de la population féminine franco-canadienne (sans le Québec) $(26,0 \%)$.

Les femmes artistes moins bien rémunérées

De façon générale, l'ensemble des femmes actives au Canada ont un revenu inférieur à celui des hommes (28 $073 \$$ pour les femmes et $43869 \$$ pour les hommes $)^{19}$. Dans la francophonie canadienne (excluant le Québec), les femmes artistes gagnent 30423 \$ et leurs confrères 43799 \$. Si l'on examine maintenant la répartition des revenus, nous observons que 44,8\% des femmes artistes franco-canadiennes (excluant le Québec) ont un revenu inférieur à $10000 \$$, près de $15 \%$ ont un revenu de 10000 à 19999 \$, 26,2 \% ont un revenu de 20000 à 49999 \$ et $13,1 \%$ ont un revenu de $50000 \$$ et plus. En somme, six femmes artistes sur dix gagnent moins de 20000 \$. L'écart entre les hommes et les femmes artistes se creuse quand le revenu atteint 50000 et plus. Enfin, si l'on place ces données dans le contexte plus général de la population active canadienne, on

18 Tableau 8, en annexe.

19 Tableau 4, en annexe. 
observe que les artistes sont proportionnellement plus nombreux (près de la moitié) à gagner moins de $20000 \$$ par année $e^{20}$.

Des variables aux hypothèses : éléments pour expliquer la minorisation des femmes artistes

En dressant le portrait sociodémographique des femmes artistes, nous venons de mettre à jour un certain nombre de caractéristiques les concernant : la féminisation de certaines disciplines artistiques comme la danse, une sous-représentation des femmes dans certaines professions artistiques comme compositrices et cheffes d'orchestre, une forte disparité entre les revenus des hommes et des femmes artistes alors que ces dernières sont plus nombreuses à posséder les diplômes les plus élevés. Mentionnons qu'en dépit de ces statistiques, il peut y avoir des femmes artistes qui soient mieux rémunérées que les hommes et/ou qui possèdent des diplômes moins élevés. Rappelons aussi que ces statistiques sont produites à partir de revenus déclarés et qu'il peut toujours y avoir un écart avec la réalité sans que cela puisse être bien documenté. Enfin, bien des raisons pourraient expliquer la disparité de revenus pour des femmes artistes qui doivent éventuellement s'acquitter de leur rôle de mère et qui feraient en sorte qu'elles soient moins engagées sur le marché du travail ou qu'elles considèrent leur revenu comme un salaire d'appoint.

Cependant, ces caractéristiques font surgir plusieurs questions relatives au statut de la femme dans les professions artistiques: est-ce que le statut de femme est un marqueur identitaire important qui conduirait à une professionnalisation différenciée entre les hommes et les femmes artistes? S'agit-il d'un processus de minorisation repérable à travers les disparités de revenu ? La féminisation d'une profession artistique entrave-t-elle les possibilités de faire carrière dans certaines disciplines ?

Nous tenterons de répondre à ces questions dans les parties suivantes, en nous intéressant tour à tour à ces marqueurs identitaires que sont les statuts de femme, d'artiste et de francophone. C'est donc par une approche déductive que nous entre-

20 Tableau 6, en annexe. 
prendrons une conceptualisation des relations de pouvoir dans les rapports linguistiques, professionnels et de sexe. Les données empiriques de notre étude ne peuvent pas constituer à elles seules ces fondements théoriques, mais les statistiques décrivant les inégalités socio-économiques entre les femmes et les hommes artistes sont assez éloquentes pour être interprétées à la lumière de ces fondements ${ }^{21}$.

Rares sont les travaux qui portent sur les femmes artistes dans la francophonie canadienne et qui adoptent une approche critique des rapports de $\operatorname{sexe}^{22}$. À la lumière de ceux réalisés sur les femmes artistes au Québec et France, nous avancerons donc plusieurs hypothèses qui pourraient nous aider à mieux comprendre les inégalités et les processus de minorisation dont ces professionnelles feraient l'objet dans le reste de la francophonie canadienne.

\section{Professionnalisation différenciée et minorisation des femmes artistes francophones : quelques hypothèses}

Par professionnalisation différenciée, nous entendons ici le processus par lequel " les fonctions, les spécialités exercées et les statuts $^{23}$ " au sein d'une même profession s'avèrent différentes selon les hommes ou les femmes. Et par minorisation des femmes artistes francophones, nous entendons le processus par lequel les statuts de femme, d'artiste et de francophone participent à une hiérarchisation des rapports sociaux au désavantage des individus

$\overline{21} \quad$ Christiane Bernier, "Femmes et ethnicité en Ontario français : limites des convergences théoriques des discours de l'identité ", Recherches féministes, vol. $8, \mathrm{n}^{\circ} 2,1995$, p. 52.

22 Une recherche avancée dans les principaux moteurs de recherche (monographies, articles scientifiques, rapports, thèses) et les publications des instituts d'étude sur les femmes au Canada (Institut des femmes d'Ottawa par exemple) montre que les travaux sur les femmes de la francophonie canadienne portent sur les représentations et les inégalités socio-économiques de ces dernières et que les travaux sur les artistes n’ont pas pour objet spécifique la francophonie hors Québec.

23 Margaret Maruani, Travail et emploi des femmes, Paris, La Découverte, 2000, citée par Hyacinthe Ravet, "Professionnalisation féminine et féminisation d'une profession : les artistes interprètes de musique ", Travail, genre et sociétés, $\mathrm{n}^{\circ}$ 9, 2003, p. 173. 
marqués par ces référents identitaires. Avant d'envisager que ces différents statuts soient cumulatifs au processus de minorisation des femmes artistes de la francophonie canadienne, nous aimerions revenir sur les enjeux d'une professionnalisation différenciée.

Pour la sociologue Hyacinthe Ravet qui a étudié précisément la professionnalisation chez les musiciennes interprètes dans les orchestres symphoniques en France, trois vagues se sont succédées dans ce processus : 1) celle des "pionnières ", 2) celle des "discrètes » et 3) celle des "paritaires ${ }^{24}$. Chaque vague correspond à des moment-clés de « l'accès de ces femmes à l'orchestre et de la construction des carrières féminines ${ }^{25}$ " que nous pourrions resituer dans un contexte plus général de l'accès des femmes aux professions qualifiées. Les "pionnières ", les " discrètes " et les " paritaires " ont chacune, à leur époque, repoussé les stéréotypes associés à leur position dans leur champ professionnel ${ }^{26}$. Les " pionnières ", en voulant prendre leur place dans l'orchestre affichaient une "volonté féministe » ou avaient, à tout le moins, conscience d'une lutte à mener. Les « discrètes » qui ont gravi les échelons de l'enseignement supérieur, à la fois comme étudiantes, puis comme enseignantes, auraient " [intériorisé] un statut problématique de la femme [...] mais doutent (ou ont douté) de leur capacité à entrer dans un orchestre ${ }^{27}$ ». Enfin, les "paritaires ", pour la plupart des étudiantes et des jeunes diplômées, ne voit pas la raison dans le fait d'être une femme qui justifierait "la nécessité de "lutter" pour faire reconnaître leurs compétences autrement qu'en concourant à jeu égal avec leurs collègues masculins ${ }^{28}$ ".

À bien des égards, cette typologie de la professionnalisation féminine des musiciennes interprètes pourrait être utilisée pour comprendre des processus similaires au Québec et dans la fran-

$\overline{24}$ Hyacinthe Ravet, "Professionnalisation féminine et féminisation d'une profession : les artistes interprètes de musique ", Travail, genre et sociétés, $\mathrm{n}^{\circ}$ 9, 2003, p. 176.

25 Ibid., p. 173.

26 Pierre Bourdieu, Les Règles de l'art. Genèse et structure du champ littéraire, Paris, Seuil, 1992.

27 Hyacinthe Ravet, op. cit., p. 173.

28 Ibid. 
cophonie canadienne au sein d'autres pratiques artistiques. D'ailleurs, l'historienne et musicologue Marie-Thérèse Lefebvre a déjà traité de la problématique de l'accès des femmes à la création musicale au Québec ${ }^{29}$ et, plus récemment, la sociologue Anna Lupien a mis à jour les rapports sociaux de sexe à travers l'analyse de la carrière d'artistes québécoises de différentes générations ${ }^{30}$. En outre, Lupien reconnaissait des caractéristiques similaires à la professionnalisation différenciée décrite par Ravet en affirmant, d'une part, que " [les] artistes femmes sont toujours moins nombreuses que leurs confrères à voir leurs œuvres présentées dans les hauts lieux de la culture ou diffusées massivement auprès du grand public ${ }^{31}$ » et, d'autre part, qu " [en] investissant de plus en plus le monde de l'art, plusieurs femmes ont $[. .$.$] introduit une présence nouvelle dans les représentations$ et remis en question les images qui les cantonnent dans des postures stéréotypées ${ }^{32}$ ".

Ainsi, si la problématique n'est pas nouvelle et qu'elle a été traitée de part et d'autre en prenant pour objet différentes disciplines artistiques selon les auteures citées précédemment, plusieurs aspects de cette problématique restent cependant à approfondir. En effet, la professionnalisation différenciée entre les hommes et les femmes ne touche pas uniquement l'accès aux professions artistiques, mais les positions et les statuts à l'intérieur de celles-ci. Par exemple, en référence à Bourdieu ${ }^{33}$, Ravet démontre que le prestige associé à certaines positions dans un orchestre symphonique, qui procurent au passage certains avantages sociaux (permanence d'emploi), agit sur la place des femmes dans l'orchestre parce que le pouvoir qui s'y exerce met en scène des rapports de force, réels et symboliques, à l'avantage des hommes. Tous ces rapports tournent autour de la possibilité de

29 Marie-Thérèse Lefebvre, La Création musicale des femmes au Québec, Montréal, Éditions du remue-ménage, 1991.

30 Anna Lupien, De la cuisine au studio, Montréal, Éditions du remue-ménage, 2012.

31 Ibid., p. 28.

32 Ibid., p. 28.

33 Pierre Bourdieu, op. cit., cité par Hyacinthe Ravet, op. cit., p. 177. 
" disposer d'une autonomie interprétative et créative versus [l'obligation] d'exécuter les décisions d'un(e) autre ${ }^{34}$ ".

Cette hiérarchisation des positions à l'intérieur d'une profession artistique peut alors être déterminante de la carrière d'une femme artiste. On comprend, en effet, que « dans un univers où les représentations du rôle de l'artiste ${ }^{35}$ [...] soudent la croyance commune dans la vocation artistique et la valeur de l'investissement dans l'activité ${ }^{36}$ ", le prestige associé à chacune des positions est tributaire de représentations sociales de la création artistique. Dans cette perspective, ces représentations valorisent la création, ou tout processus créatif qui concède une grande autonomie à l'artiste, alors qu'à l'inverse, toute approche techniciste et utilitaire de la création est dévalorisée. C'est d'ailleurs sur ces représentations que s'attarde le sociologue Pierre-Michel Menger pour expliquer que l'artiste constitue la « figure exemplaire du travailleur ${ }^{37}$ " étant donné les caractéristiques suivantes de sa profession : " fort degré d'engagement dans l'activité, autonomie élevée dans le travail, flexibilité acceptée voire revendiquée, arbitrages risqués entre gains matériels et gratifications souvent non monétaires, exploitation stratégique des manifestations inégalitaires du talent ${ }^{38} »$.

Autrement dit, en comparant les professions artistiques à tout autre domaine professionnel, il en vient à la conclusion que, dans le jeu de la concurrence propre au marché du travail, l'artiste est celui qui est le plus apte à composer avec l'incertitude de son activité professionnelle et à exploiter son propre talent. Par conséquent, selon la perspective de Menger, et s'il y a une professionnalisation différenciée entre les hommes et les femmes artistes, on peut alors s'interroger sur la façon dont les femmes artistes tirent leur épingle du jeu. Sont-elles aussi compétitives

\footnotetext{
34 Hyacinthe Ravet, op. cit., p. 182.

35 Représentations qui s'apparentent parfois au mythe du " génie artistique ".

36 Hyacinthe Ravet, op. cit., p. 182.

37 Pierre-Michel Menger, Portrait de l'artiste en travailleur. Métamorphoses du capitalisme, Paris, Seuil, coll. "La République des idées ", 2002, p. 8.

38 Pierre-Michel Menger, Le Travail créateur. S'accomplir dans l'incertain, Paris, Gallimard/Seuil, 2009, p. 9.
} 
que les hommes ou, plutôt, leurs stratégies de carrière sont-elles aussi bien établies et reçues que celles de leurs confrères ? Quels sont les dispositifs ou les circonstances qui favorisent leur professionnalisation et leur réussite ? Menger distingue, à cet égard, quatre points dont dépend l'artiste quant à sa réussite. Le premier est relatif à l'artiste lui-même et à sa volonté de faire carrière; le second est lié à " l'environnement de son activité et des conditions (matérielles, juridiques, politiques) dans lesquelles son travail est entrepris $^{39}$ "; le troisième renvoie à la " qualité du travail de l'équipe qui s'affaire dans le projet échafaudé pour créer une œuvre ou un spectacle ${ }^{40}$ » et, enfin, le quatrième concerne "l'évaluation de ceux, pairs, professionnels, consommateurs profanes, qui reçoivent l'œuvre achevée ${ }^{41} »$. Ainsi, la garantie du succès qui démarque les artistes entre eux repose sur la volonté d'autres acteurs et la mise en ouvre d'autres processus. Les représentations et les attentes qui en découlent ne sont certainement pas exemptes de représentations sexuées du travail.

Par ailleurs, un autre processus, celui-là lié à l'évolution des disciplines artistiques, est également à examiner. Par exemple, devant le déclin de l'écoute de la musique "classique " ou l'engouement pour les arts numériques, on ne peut que constater que le statut de certaines disciplines artistiques et de certains champs qui les composent change. Ces changements peuvent conduire à une dévalorisation de certaines professions artistiques liée à la perte de prestige de la discipline ou du champ artistique en question. C'est ce que décrit notamment Ravet au sujet des orchestres symphoniques. Elle observe, entre autres, que les actions de médiation culturelle pour favoriser l'écoute de la " musique classique " ouvrent la voie à diverses interprétations sur la valeur même de cette musique et de leurs interprètes qui y décèlent soit une perte du statut d'exception acquis au fil du temps et confirmé par la fréquentation d'un public averti, soit une ouverture à l'éducation d'un public plus large auquel il faut

\begin{tabular}{ll}
\hline 39 & Ibid., p. 9. \\
40 & Ibid., p. 9. \\
41 & Ibid., p. 9.
\end{tabular}


nécessairement s'adapter. Nous avions observé un phénomène similaire à travers la mise en scène de la Symphonie du Millénaire sur le site de l'Oratoire Saint-Joseph à Montréal ${ }^{42}$. En effet, pour rapprocher le public de la musique contemporaine, ou pour l'y initier, les organisateurs de l'événement avaient imaginé un processus participatif. Une partie du public contribuait à la Symphonie en faisant teinter des cloches. Le choix du lieu du concert faisait également partie de la stratégie pour attirer un plus large public. Ainsi, au fur et à mesure que se produisent une professionnalisation des femmes artistes et une féminisation de certaines pratiques artistiques, on assiste également à une déqualification de ces pratiques qui, pour certains acteurs les moins ouverts à la parité, sont trois processus concomitants.

En somme, et bien que notre recherche sur les artistes de la francophonie canadienne ne nous renseigne pas entièrement sur ces divers processus, les données montrant que les femmes artistes sont moins rémunérées nous laissent au moins entrevoir que divers mécanismes sont probablement marqués par une division sexuée du travail. Ce qui oriente éventuellement les femmes artistes vers des carrières moins prestigieuses où elles ont moins l'opportunité d'exercer leur pouvoir et de tirer des avantages pécuniaires de leur profession. D'autres facteurs peuvent venir accentuer la difficulté des femmes à faire carrière comme artiste. Le contexte social peut exercer certaines contraintes sur la profession artistique comme cela semble être le cas dans la francophonie canadienne.

\section{Les conditions de pratique des artistes francophones au Canada}

Dans les parties précédentes, nous avons décrit les caractéristiques sociodémographiques des femmes artistes de la francophonie canadienne et nous avons soulevé plusieurs questions liées à la professionnalisation différenciée des pratiques artistiques entre

$\overline{42}$ Anne Robineau, "Les Stratégies sociales des artistes contemporains dans l'utilisation du sacré. Le cas de la Symphonie du Millénaire ", Religiologiques, Montréal, Université du Québec à Montréal, n 22, 2000, p. 85-99. 
les hommes et les femmes. Nous avions émis l'hypothèse d'une triple minorisation du statut de femme, d'artiste et de francophone. Nous aimerions maintenant cerner davantage les conditions de pratique des artistes de la francophonie canadienne afin de comprendre si certaines de ces conditions constituent un frein aux stratégies de carrière des artistes, y compris les femmes artistes. Auquel cas, nous pourrions évoquer l'hypothèse d'une minorisation liée à l'appartenance francophone hors Québec.

Les conditions de pratique auxquelles nous faisons référence représentent l'ensemble des structures, des organismes, des dispositifs et des réseaux qui participent à la création, la production, la diffusion et la réception des œuvres et des produits culturels des artistes franco-canadiens. Nous ne pouvons que brièvement ici en faire l'inventaire et confirmer que, comme partout ailleurs au Canada, le milieu des arts et de la culture de la francophonie canadienne s'est fortement institutionnalisé et professionnalisé au cours des dernières décennies. On en veut pour preuve l'augmentation du nombre d'organismes artistiques et culturels voués à la diffusion des œuvres créées ou produites par les artistes franco-canadiens $s^{43}$, l'existence d'une fédération nationale regroupant la plupart de ces organismes ${ }^{44}$, la mise sur pied de réseaux de diffusion des arts de la scène organisés par " aire géographique »

43 Par exemple : Alliance culturelle de l'Ontario (ACO); Association acadienne des artistes professionnel.le.s du Nouveau-Brunswick (AAAPNB); Association culturelle franco-manitobaine (ACFM); Association franco-yukonnaise (AFY); Centre culturel franco-manitobain (CCFM); Conseil culturel et artistique francophone de la Colombie-Britannique (CCAFCB); Conseil culturel fransaskois (CCF); Conseil provincial des sociétés culturelles (CPSC); Fédération culturelle acadienne de la Nouvelle-Écosse (FéCANE); Fédération culturelle de l'Île-du-Prince-Édouard (FCIPE); Fédération des francophones de Terre-Neuve et du Labrador (FFTNL); Fédération franco-ténoise (FFT); Regroupement artistique francophone de l'Alberta (RAFA); Alliance des producteurs francophones du Canada (APFC); Alliance nationale de l'industrie musicale (ANIM); Association des groupes en arts visuels francophones (AGAVF); Association des théâtres francophones du Canada (ATFC); Front des réalisateurs indépendants du Canada (FRIC); Regroupement des éditeurs canadiens-français (RÉCF); Réseau national des galas et de la chanson (RNGC).

44 La Fédération culturelle canadienne-française. 
au $\operatorname{Canada}^{45}$, la remise de prix récompensant les artistes, une critique plus régulière des œuvres et des artistes grâce à l'existence de médias, de revues spécialisées et d'une présence plus accrue sur le web ainsi qu'une reconnaissance de plusieurs artistes à l'extérieur de leur communauté d'origine, notamment sur la scène québécoise et internationale. Enfin, la création de programmes d'enseignement, bien qu'encore insuffisants au sein de la francophonie canadienne, aux niveaux collégial et universitaire, dans certaines disciplines artistiques (théâtre, musique, arts visuels, littérature) a contribué à la professionnalisation des artistes et des travailleurs culturels.

Tout cela ne pourrait pas se développer sans un apport financier des gouvernements provinciaux, territoriaux et fédéral ainsi que de celui des municipalités. À ce titre, il faut aussi rappeler que, dans certains cas, et surtout depuis la mise en œuvre de la Loi sur les langues officielles au Canada et de l'adoption de législations linguistiques au niveau des provinces et des territoires, des enveloppes budgétaires sont prévues pour le développement des communautés de langue officielle en situation minoritaire ${ }^{46}$.

45 Réseau atlantique de diffusion des arts de la scène (RADARTS); Réseau des grands espaces (RGE); Réseau Ontario (RO); Scènes francophones.

46 Par exemple, il existe une entente multipartite entre l'organisme national culturel francophone et des agences fédérales pour la culture : "L'Entente pour le développement des arts et de la culture de la francophonie canadienne a été conclue le 14 septembre 2009 entre la FFCF et le Ministère du Patrimoine canadien, le Centre national des arts, le Conseil des arts du Canada, l'Office national du film du Canada, la Société Radio-Canada et Téléfilm Canada. Elle fait suite à une première entente, l'Entente multipartite sur la culture, signée en 1998 et qui était arrivée à échéance le 31 mars 2008. Le but était de favoriser les conditions de développement des arts et de la culture chez les communautés francophones en situation minoritaire à travers des collaborations, des actions concertées, un appui à la recherche et la prise en compte du dialogue interculturel et de la dualité linguistique. Les différentes institutions signataires de l'Entente se sont donc engagées à concentrer leurs efforts autour des enjeux suivants : "le soutien à la relève et au développement professionnel; le renforcement des structures culturelles et artistiques; l'accroissement de la visibilité et du rayonnement des artistes; le renouvellement du public; l'intégration des nouvelles technologies" ". Entente pour le développement des arts et de la culture de la francophonie canadienne, 2009, cité par Robineau, Un Regard actuel sur la situation des artistes dans la francophonie canadienne, op. cit. 
Un certain nombre de projets culturels et d'activités artistiques sont financés par cet intermédiaire, même si la plupart des divers budgets destinés aux communautés francophones sont absorbés par d'autres secteurs comme l'éducation ou la santé, à l'instar d'autres collectivités au Canada. Le secteur éducatif réserve également du financement pour des activités pédagogiques liées à l'enseignement des arts et à des sorties culturelles. De façon générale, les politiques culturelles des provinces et des territoires ainsi que celles émises par des municipalités orientent l'administration des ressources pour le développement culturel de leurs collectivités respectives; ressources auxquels les organismes artistiques et culturels francophones peuvent prétendre ${ }^{47}$.

Ce bref aperçu de l'institutionnalisation et la professionnalisation des arts et de la culture au sein de la francophonie canadienne témoigne d'un dynamisme des communautés francophones, mais bien des défis subsistent pour que les artistes puissent tous réussir et être soutenus à la hauteur de leurs attentes. En effet, toutes les conditions ne sont pas toujours réunies de façon optimale, selon les quatre points développés par Menger, pour qu'un artiste développe sa carrière.

Parmi ces défis, il y a la nécessité de composer avec une densité faible de la population francophone et francophile dans certaines régions. Cela influence la taille du réseau professionnel local des arts, de la culture et du public. Et faute d'une masse critique, les ressources en termes de formation en français, d'aide à la création et à la production ainsi qu'à la diffusion des arts peuvent être insuffisantes pour développer des projets culturels, des activités artistiques et soutenir les artistes ${ }^{48}$. Les organismes culturels et artistiques francophones doivent aussi redoubler d'efforts pour sensibiliser certains acteurs à l'extérieur de leur communauté

\footnotetext{
$47 \quad$ Monica Gattinger et Diane Saint-Pierre (dir.), Les Politiques culturelles des gouvernements provinciaux et territoriaux du Canada, Québec, Presses de l'Université Laval, coll. "Gouvernance et gestion publique », 2011.

Lucie Hotte, "Entre l'esthétique et l'identité : la création en contexte minoritaire ", dans Joseph Yvon Thériault, Anne Gilbert et Linda Cardinal (dir.), L'Espace francophone en milieu minoritaire au Canada : nouveaux enjeux, nouvelles mobilisations, Québec, Fides, 2008, p. 334.
} 
linguistique qui pourraient contribuer au développement de la scène artistique francophone dans leur province ou leur territoire. Enfin, la question du public rejoint d'autres enjeux, ceux de la réception et de la reconnaissance du travail artistique, de la formation d'une critique (pairs, chercheurs, experts, commissaires, etc.) qui participent à la légitimation des œuvres et des artistes et de la constitution d'un patrimoine artistique. À certains égards, ces problématiques s'apparentent à ce que peuvent vivre des artistes en région au Québec et au Canada où, comme le note la sociologue Andrée Fortin, les acteurs culturels peuvent occuper plusieurs rôles : " artistes, critiques, diffuseurs ${ }^{49}$ ".

Un autre défi, et non le moindre, est l'ambiguïté du rôle de l'artiste en milieu minoritaire francophone qui est souvent tiraillé entre son statut d'artiste et son appartenance à la communauté francophone. En effet, de par leur situation linguistique minoritaire, les communautés francophones sont engagées dans un processus constant de revitalisation linguistique et culturelle. Les arts et la culture sont considérés comme un élément stratégique de cette revitalisation et du développement des communautés ${ }^{50}$. Cette approche du développement communautaire en termes de vitalité est assez bien documentée. Le sociologue Éric Forgues, qui proposait récemment un cadre conceptuel s'appuyant sur les concepts d'autonomie, de vitalité et d'identité, recensait une partie des travaux sur la question, travaux qui renvoyaient, certes, aux aspects démographiques de ces communautés, mais aussi au statut de la langue et au soutien de leurs institutions ${ }^{51}$.

49 Andrée Fortin, "Présentation », dans Andrée Fortin (dir.), Produire la culture, produire l'identité?, Québec, Presses de l'Université Laval, 2000, p. XVII.

50 Voir par exemple: Anne Robineau, "Les États généraux des arts et de la culture de la communauté acadienne du Nouveau-Brunswick: un outil de développement ", dans Claudine Audet et Diane Saint-Pierre (dir.), Tendances et défis des politiques culturelles dans les pays occidentaux, Québec, Presses de l'Université Laval, coll. "Chaire Fernand-Dumont sur la culture ", 2009, p. 201-216; Association acadienne des artistes professionnel.le.s du NouveauBrunswick, Stratégie globale pour l'intégration des arts et de la culture dans la société acadienne au Nouveau-Brunswick, Moncton, 2009, [en ligne] http:// www.aaapnb.ca/media_uploads/pdf/6404.pdf, consulté le 30 mars 2011.

51 Éric Forgues, "Autonomie, vitalité et identité des communautés en situation minoritaire. Proposition d'un cadre conceptuel ", International Journal of 
Cependant, selon Lucie Hotte, le discours sur la vitalité et l'identité des communautés francophones peut faire jouer un rôle assez réducteur aux artistes, aux institutions et aux divers intervenants culturels s'il est systématiquement associé à une sorte de " missionnariat » où tout est mobilisé pour «valoriser la culture du groupe ${ }^{52}$ " que certains sociologues qualifient $d$ ' " art du minoritaire ${ }^{53}$ ». Cette tendance semble s'amoindrir au fil du temps avec la professionnalisation du milieu artistique francocanadien qui procure aux artistes les moyens de comparer leurs œuvres avec celles créées ailleurs. Cependant, les nouvelles actions d'animation et de médiation culturelles ${ }^{54}$ qui promeuvent l'éducation et la sensibilisation du public à l'art et que l'on multiplie à travers divers initiatives et programmes ${ }^{55}$, sont susceptibles, malgré leur bien-fondé, d'amalgamer à nouveau le travail des artistes à cette production de l'identité francophone, autrement nommée la "construction identitaire ».

Ainsi, les conditions de pratique des artistes de la francophonie canadienne sont soumises à différentes pressions sociales, individuelles, politiques et économiques dont il est conviendrait d'en

Canadian Studies / Revue internationale d'études canadiennes, n 42, 2010, p. 188.

52 Lucie Hotte, op. cit., p. 320.

53 Jean Lafontant, "L'Art “minoritaire”, entre les conventions identitaires et l'émancipation ", dans Robert Dickson, Annette Ribordy et Micheline Tremblay (dir.), Toutes les photos finissent-elles par se ressembler?, Actes du forum sur la situation des arts au Canada français, Sudbury, Prise de parole, 1999, p. 43-49, cité par Lucie Hotte, op. cit., p. 321.

54 Jean-Marie Lafortune (dir.), La Médiation culturelle : le sens des mots et l'essence des pratiques, Sainte-Foy, Presses de l'Université du Québec, coll. «Culture et publics ", 2012.

55 On peut penser au guide d'intervention en médiation culturelle et artistique destiné au secteur éducatif des francophones hors Québec et qui fait le lien entre éducation artistique et construction de l'identité francophone : « Le passeur culturel accompagne la personne, l'élève, l'enfant ou l'adulte, dans la construction de son identité culturelle en créant des occasions signifiantes de découverte et d'expression de la culture francophone tout en étant ouvert sur les autres cultures. Par des interventions qui éveillent les sentiments d'appartenance, de compétence et d'autonomie, le passeur culturel encourage une démarche de réflexion sur le rapport à soi, le rapport à l'autre et le rapport à l'environnement ». Extrait du site web de la Trousse du passeur culturel, [en ligne] http://www.passeurculturel.ca/, consulté le 15 janvier 2013. 
étudier plus profondément les dynamiques. Car, de manière générale, ces dynamiques nous renseignent à la fois sur le contexte et la façon dont les ouvres sont produites, mais aussi sur leur impact sur l'imaginaire social. Elles participent alors à " un processus [...] jamais achevé d'attribution de sens; processus d'appropriation dont le résultat est que les œuvres sont ce qu'on en fait et qu'il ne suffit pas d'en analyser les propriétés formelles pour en saisir la portée ${ }^{56} \%$.

L'analyse des pratiques artistiques dans la francophonie canadienne représente donc un travail complexe qui dépasse le cadre de cet article. Cependant, une analyse plus complète pourrait nous aider à conclure avec encore plus de certitude qu'il y a une minorisation des artistes à cause de leur appartenance à un contexte linguistique francophone minoritaire. Car force est d'admettre que plusieurs difficultés sont inhérentes à ce contexte. En effet, ces communautés, pour les raisons évoquées plus haut, possèdent des ressources plus restreintes. Ce qui fait en sorte que les artistes éprouvent de la difficulté à créer ou produire dans leur communauté et à intégrer d'autres pôles artistiques, notamment ceux de la francophonie nationale et internationale et ceux de la majorité anglophone. Cette intégration à des réseaux nationaux et internationaux est pourtant une condition sine qua non à une plus grande reconnaissance de leur travail. Autrement dit, dans le jeu de concurrence tel que le décrit Menger, les artistes francophones en situation minoritaire peuvent se trouver plus souvent dans des situations d'inégalités. Et si, de surcroît, il existe un processus de professionnalisation différenciée tel que le décrit Ravet, il semble plus que probable que les femmes artistes en soient les grandes perdantes.

\section{Conclusion}

Dans cet article, nous nous proposions d'examiner le lien entre des données statistiques montrant plusieurs inégalités entre les femmes et les hommes artistes dans la francophonie canadienne et des processus de minorisation liés aux statuts de femme,

56 Andrée Fortin, op. cit., p. XVII. 
d'artiste et de francophone. Si la première partie de notre démonstration a exposé en quoi, statistiquement, ces inégalités consistaient (féminisation de certaines disciplines artistiques, détention des diplômes les plus élevés chez les femmes, rémunération moindre de leur activité professionnelle), la deuxième partie a plutôt avancé plusieurs hypothèses relatives à une éventuelle minorisation des femmes artistes de la francophonie canadienne. Ces hypothèses reposaient en grande partie sur l'idée d'une professionnalisation différenciée qui se fait aux dépens des femmes artistes. Il a été intéressant d'exposer la façon dont certaines représentations sociales sur le genre sont intériorisées au point de déterminer la position et le statut d'artistes professionnels au sein d'un même corps de métier, voire de déqualifier certaines pratiques artistiques au fur et à mesure que des professions artistiques se féminisent. Cette démonstration n'a pas pu être directement mise en lien avec nos données statistiques sur les femmes artistes de la francophonie canadienne, mais elle a contribué à poser la question de la hiérarchisation des pratiques et d'une division sexuée du travail artistique.

La troisième partie a aussi décrit les conditions de pratique dans le contexte minoritaire francophone. Ce contexte peut parfois freiner l'expansion d'une carrière artistique selon les contraintes et les pressions sociales exercées sur l'artiste. Nous avons, entre autres, souligné, le manque de ressources en français, des aspects démographiques qui ont un impact autant sur la taille du public que sur les infrastructures de diffusion et sur la critique des œuvres et des artistes. Puis nous avons évoqué la tension entre les aspirations des artistes à une reconnaissance fondée sur la qualité de leurs œuvres et une perspective identitaire qui valorise plutôt leur carrière professionnelle à travers leur appartenance à une communauté linguistique. Les contraintes de la création artistique en milieu minoritaire peuvent alors s'ajouter à celles provoquées par une division sexuée du travail, y compris dans la sphère artistique.

Ainsi, l'articulation des hypothèses énoncées tout au long de ce texte a expliqué en partie les inégalités qui ressortaient dans la 
description des données statistiques. Cet exercice a également permis de mettre à jour une perspective peu étudiée sur un objet rarement analysé : celle des rapports sexués du travail chez les artistes professionnelles de la francophonie canadienne. On peut dès lors se poser les questions suivantes : les femmes artistes seraient-elles plus nombreuses encore à choisir cette profession si elle comportait moins d'obstacles réels et symboliques vers la réussite de leur carrière? Quel en serait l'impact sur la francophonie canadienne et son patrimoine artistique? Comment ne pas penser au rôle positif sur l'identité collective de toutes ces communautés francophones en situation minoritaire si ces femmes artistes avaient davantage l'opportunité de s'exprimer par leurs pratiques? Pour passer de "pionnières " à "paritaires ", il reste donc du travail à faire.

\section{Bibliographie}

Arbour, Rose-Marie, "Le Cercle des automatistes et la différence des femmes ", Études françaises, vol. 34, nos 2-3, 1998, p. 157-173.

Association acadienne des artistes professionnel.le.s du Nouveau-Brunswick, Stratégie globale pour l'intégration des arts et de la culture dans la société acadienne au Nouveau-Brunswick, Moncton, 2009, [en ligne] http:// www.aaapnb.ca/media_uploads/pdf/6404.pdf, consulté le 30 mars 2011).

Bernier, Christiane, «Femmes et ethnicité en Ontario français : limites des convergences théoriques des discours de l'identité ", Recherches féministes, vol. 8, no 2, 1995, p. 47-66.

Bourdieu, Pierre, Les Règles de l'art. Genèse et structure du champ littéraire, Paris, Seuil, 1992.

Descarries, Francine, Chronologie de l'histoire des fermmes au Québec et rappel d'événements marquants à travers le monde, 2007, [en ligne] http://www.unites.uqam.ca/arir/pdf/chronologieNouvelleVersionJuin2007.pdf, consulté le 15 janvier 2013. 
Descarries, Francine, "Le Mouvement des femmes et ses courants de pensée: essai de typologie ", Les Documents de l'ICREF, n 19, Ottawa, Institut canadien de recherches sur les femmes, 1998.

Fidecaro, Agnese et Stéphanie Lachat (dir.), Profession: créatrice. La place des femmes dans le champ artistique, Actes du colloque de l'Université de Genève, 18 et 19 juin 2004, Lausanne, Antipodes, 2007.

Forgues, Éric, "Autonomie, vitalité et identité des communautés en situation minoritaire. Proposition d'un cadre conceptuel », International Journal of Canadian Studies / Revue internationale d'études canadiennes, no 42, 2010, p. 183-206.

Fortin, Andrée, "Présentation ", dans Andrée Fortin (dir.), Produire la culture, produire l'identité?, Québec, Presses de l'Université Laval, 2000, p. I-XIII.

Gattinger, Monica et Diane Saint-Pierre (dir.), Les Politiques culturelles des gouvernements provinciaux et territoriaux du Canada, Québec, Presses de l'Université Laval, coll. "Gouvernance et gestion publique », 2011.

Hotte, Lucie, "Entre l'esthétique et l'identité : la création en contexte minoritaire», dans Joseph Yvon Thériault, Anne Gilbert et Linda Cardinal (dir.), L'Espace francophone en milieu minoritaire au Canada : nouveaux enjeux, nouvelles mobilisations, Québec, Fides, 2008, p. 319350.

Kergoat, Danièle, "Rapports sociaux et division du travail entre les sexes ", dans Margaret Maruani (dir.), Femmes, genre et sociétés, Paris, La Découverte, 2005, p. 94-101.

Kergoat, Danièle, «Division sexuelle du travail et rapports sociaux de sexe », dans Helena Hirata, Françoise Laborie, Hélène Le Doaré et Danièle Senotier (dir.), Dictionnaire critique du féminisme, Paris, Presses universitaires de France, 2000, p. 35-44.

Lafontant, Jean, «L'Art "minoritaire", entre les conventions identitaires et l'émancipation ", dans Robert Dickson, Annette Ribordy et Micheline Tremblay (dir.), Toutes les photos finissent-elles par se ressembler?, Actes du forum sur la situation des arts au Canada français, Sudbury, Prise de parole, 1999, p. 43-49.

Lafortune, Jean-Marie (dir.), La Médiation culturelle : le sens des mots et l'essence des pratiques, Sainte-Foy, Presses de l'Université du Québec, coll. "Culture et publics », 2012.

Lefebvre, Marie-Thérèse, La Création musicale des femmes au Québec, Montréal, Éditions du remue-ménage, 1991.

Lupien, Anna, De la cuisine au studio, Montréal, Éditions du remueménage, 2012. 
Maruani, Margaret, Travail et emploi des femmes, Paris, La Découverte, 2000.

Menger, Pierre-Michel, Le Travail créateur. S'accomplir dans l'incertain, Paris, Gallimard/Seuil, 2009.

Menger, Pierre-Michel, Portrait de l'artiste en travailleur. Métamorphoses du capitalisme, Paris, Seuil, coll. " La République des idées », 2002.

Ravet, Hacinthe, "Professionnalisation féminine et féminisation d'une profession : les artistes interprètes de musique ", Travail, genre et sociétés, $\mathrm{n}^{\circ}$ 9, 2003, p. 173-195.

Robineau, Anne, "Les Stratégies sociales des artistes contemporains dans l'utilisation du sacré. Le cas de la Symphonie du Millénaire ", Religiologiques, Montréal, Université du Québec à Montréal, $\mathrm{n}^{\circ}$ 22, 2000, p. 85-99.

Robineau, Anne, Un Regard actuel sur la situation des artistes dans la francophonie canadienne, Rapport de recherche pour le Conseil des arts du Canada, Moncton, Institut canadien de recherche sur les minorités linguistiques (2013, à paraître).

Robineau, Anne, "Les États généraux des arts et de la culture de la communauté acadienne du Nouveau-Brunswick: un outil de développement ", dans Claudine Audet et Diane Saint-Pierre (dir.), Tendances et défis des politiques culturelles dans les pays occidentaux, Québec, Presses de l'Université Laval, coll. "Chaire Fernand-Dumont sur la culture ", 2009, p. 201-216.

Robineau, Anne, "Musique, femme et création musicale ", dans Dire, Université de Montréal, vol. 12, nº ${ }^{\circ}$ janvier 2004, p. 19-22.

Statistique Canada, Femmes au Canada : rapport statistique fondé sur le sexe, 2010-2011, Sixième édition, 89-503-XIF, Ottawa, 2011.

Trousse du passeur culturel, [en ligne] http://www.passeurculturel.ca/, consulté le 15 janvier 2013. 


\section{Annexe}

Tableau 1: nombre et pourcentage d'artistes francophones, hommes et femmes, hors Québec et au Canada par rapport au nombre d'artistes canadiens

\begin{tabular}{|l|l|c|c|}
\hline & \multicolumn{1}{|c|}{ Sexe } & Nombre & \multicolumn{1}{c|}{$\%$} \\
\hline Artistes au Canada & Total & 119045 & $100,0 \%$ \\
\cline { 2 - 4 } & hommes & 57990 & $100,0 \%$ \\
\cline { 2 - 4 } & femmes & 61060 & $100,0 \%$ \\
\hline \multirow{4}{*}{ Artistes francophones au Canada } & Total & 23655 & $19,9 \%$ \\
\cline { 2 - 4 } & hommes & 12350 & $21,3 \%$ \\
\cline { 2 - 4 } & femmes & 11310 & $18,5 \%$ \\
\hline \multirow{3}{*}{$\begin{array}{l}\text { Artistes francophones au Canada moins } \\
\text { le Québec }\end{array}$} & Total & 3125 & $2,6 \%$ \\
\cline { 2 - 4 } & hommes & 1415 & $2,4 \%$ \\
\cline { 2 - 4 } & femmes & 1715 & $2,8 \%$ \\
\hline
\end{tabular}

Source : Équipe de recherche, Programme d'appui aux langues officielles, Patrimoine canadien, basée sur les données du recensement du Canada, Statistique Canada, 2006, échantillonnage de $20 \%$. Population de 15 ans et plus déclarant un revenu.

Tableau 2: nombre et pourcentage d'artistes, hommes et femmes, francophones hors Québec et au Canada par rapport à la population francophone active hors Québec, la population francophone active au Canada et la population active totale au Canada

\begin{tabular}{|l|l|c|c|c|}
\hline \multicolumn{1}{|c|}{ Région } & \multicolumn{1}{|c|}{ Sexe } & Artistes & $\begin{array}{c}\text { Population } \\
\text { active }\end{array}$ & $\begin{array}{c}\% \text { de la } \\
\text { population active }\end{array}$ \\
\hline Population au Canada & Total & 119045 & 18201265 & $0,65 \%$ \\
\cline { 2 - 5 } & hommes & 57990 & 9480555 & $0,61 \%$ \\
\cline { 2 - 5 } & femmes & 61060 & 8720710 & $0,70 \%$ \\
\hline Francophones au Canada & Total & 23655 & 4258720 & $0,56 \%$ \\
\cline { 2 - 5 } & hommes & 12350 & 2228575 & $0,55 \%$ \\
\cline { 2 - 5 } & femmes & 11310 & 2030143 & $0,56 \%$ \\
\hline \multirow{2}{*}{$\begin{array}{l}\text { Francophones au Canada } \\
\text { moins le Québec }\end{array}$} & Total & 3125 & 603338 & $0,52 \%$ \\
\cline { 2 - 5 } & hommes & 1415 & 308465 & $0,46 \%$ \\
\cline { 2 - 5 } & femmes & 1715 & 294868 & $0,58 \%$ \\
\hline $\begin{array}{l}\text { Source : Équipe de recherche, Programme d'appui aux langues officielles, Patrimoine canadien, basée sur les } \\
\text { donnés du recensement du Canada, Statistique Canada, 2006, échantillonnage de 20 \%. Population de 15 ans et } \\
\text { plus déclarant un revenu. }\end{array}$
\end{tabular}




\begin{tabular}{|l|l|c|}
\hline \multicolumn{2}{|l|}{ Tableau 3: Revenu des artistes, hommes et femmes, francophones hors Québec et au } \\
Canada par rapport au revenu de la population francophone hors Québec, la population \\
francophone au Canada et la population totale au Canada \\
\hline \multirow{4}{*}{ Population au Canada } & \multicolumn{1}{|c|}{ Sexe } & Revenu moyen \\
\hline & Total & $\$ 24029$ \\
\cline { 2 - 3 } & hommes & $\$ 27674$ \\
\cline { 2 - 3 } & femmes & $\$ 20567$ \\
\hline Francophones au Canada & Total & $\$ 25627$ \\
\cline { 2 - 3 } & hommes & $\$ 29031$ \\
\cline { 2 - 3 } & femmes & $\$ 21898$ \\
\hline Francophones au Canada moins le Québec & Total & $\$ 24993$ \\
\cline { 2 - 3 } & hommes & $\$ 29265$ \\
\cline { 2 - 3 } & femmes & $\$ 21388$ \\
\hline $\begin{array}{l}\text { Source : Équipe de recherche, Programme d'appui aux langues officielles, Patrimoine canadien, basée sur les } \\
\text { données du recensement du Canada, Statistique Canada, 2006, échantillonnage de 20\%. Population de } 15 \text { ans et } \\
\text { plus déclarant un revenu. }\end{array}$ & \\
\hline
\end{tabular}

Tableau 4 : Pourcentage du revenu des artistes, hommes et femmes, francophones hors Québec et écart de revenu moyen par rapport à l'ensemble de la population francophone hors Québec

\begin{tabular}{|l|l|l|c|c|c|}
\hline \multicolumn{1}{|c|}{ région } & \multicolumn{1}{|c|}{ sexe } & artistes & $\begin{array}{c}\text { Population } \\
\text { active }\end{array}$ & $\begin{array}{c}\% \text { du } \\
\text { revenu de } \\
\text { la } \\
\text { population } \\
\text { active } \\
\text { totale }\end{array}$ & $\begin{array}{c}\text { Écart du revenu } \\
\text { moyen de la } \\
\text { population } \\
\text { active totale }\end{array}$ \\
\hline \multirow{2}{*}{ Canada } & Total & $\$ 24029$ & $\$ 36301$ & $66,2 \%$ & $\$ 12272$ \\
\cline { 2 - 6 } & hommes & $\$ 27674$ & $\$ 43869$ & $63,1 \%$ & $\$ 16195$ \\
\cline { 2 - 6 } & femmes & $\$ 20567$ & $\$ 28073$ & $73,3 \%$ & $\$ 7506$ \\
\hline \multirow{2}{*}{$\begin{array}{l}\text { Cancophones au } \\
\text { Canada }\end{array}$} & Total & $\$ 25627$ & $\$ 33153$ & $77,3 \%$ & $\$ 7526$ \\
\cline { 2 - 6 } & hommes & $\$ 29031$ & $\$ 38897$ & $74,6 \%$ & $\$ 9866$ \\
\cline { 2 - 6 } & femmes & $\$ 21898$ & $\$ 26860$ & $81,5 \%$ & $\$ 4962$ \\
\hline $\begin{array}{l}\text { Francophones au } \\
\text { Québec }\end{array}$ & Total & $\$ 24993$ & $\$ 37270$ & $67,1 \%$ & $\$ 12277$ \\
\cline { 2 - 6 } & hommes & $\$ 29265$ & $\$ 43799$ & $66,8 \%$ & $\$ 14534$ \\
\cline { 2 - 6 } & femmes & $\$ 21388$ & $\$ 30423$ & $70,3 \%$ & $\$ 9035$ \\
\hline
\end{tabular}

Source : Équipe de recherche, Programme d'appui aux langues officielles, Patrimoine canadien, basée sur les données du recensement du Canada, Statistique Canada, 2006, échantillonnage de $20 \%$. Population de 15 ans et plus déclarant un revenu. 


\begin{tabular}{|c|c|c|c|c|c|c|c|}
\hline Groupe & Sexe & $\begin{array}{l}\text { Tranche de } \\
\text { revenu }\end{array}$ & avec revenu & $\begin{array}{l}\text { moins que } \\
10000 \$\end{array}$ & $\begin{array}{l}10000 \\
\text { à } 19999 \$\end{array}$ & $\begin{array}{c}20000 \\
\text { à } 49999 \$\end{array}$ & $\begin{array}{l}50000 \$ \\
\text { et plus }\end{array}$ \\
\hline \multirow[t]{3}{*}{ Artistes au Canada } & Total & nombre & 119045 & 49415 & 22435 & 29735 & 17485 \\
\hline & hommes & nombre & 57990 & 21115 & 10910 & 15295 & 10675 \\
\hline & femmes & nombre & 61060 & 28310 & 11530 & 14430 & 6810 \\
\hline \multirow{3}{*}{$\begin{array}{l}\text { Artistes francophones au } \\
\text { Canada }\end{array}$} & Total & nombre & 23655 & 8850 & 4788 & 6158 & 3858 \\
\hline & hommes & nombre & 12350 & 4023 & 2575 & 3378 & 2363 \\
\hline & femmes & nombre & 11310 & 4833 & 2200 & 2765 & 1503 \\
\hline \multirow{3}{*}{$\begin{array}{l}\text { Artistes francophones au } \\
\text { Canada moins le Québec }\end{array}$} & Total & nombre & 3125 & 1263 & 505 & 835 & 545 \\
\hline & hommes & nombre & 1415 & 505 & 238 & 383 & 310 \\
\hline & femmes & nombre & 1715 & 768 & 255 & 450 & 225 \\
\hline \multirow[t]{3}{*}{ Artistes au Canada } & Total & $\%$ & $100,0 \%$ & $41,5 \%$ & $18,8 \%$ & $25,0 \%$ & $14,7 \%$ \\
\hline & hommes & $\%$ & $100,0 \%$ & $36,4 \%$ & $18,8 \%$ & $26,4 \%$ & $18,4 \%$ \\
\hline & femmes & $\%$ & $100,0 \%$ & $46,4 \%$ & $18,9 \%$ & $23,6 \%$ & $11,2 \%$ \\
\hline \multirow{3}{*}{$\begin{array}{l}\text { Artistes francophones au } \\
\text { Canada }\end{array}$} & Total & $\%$ & $100,0 \%$ & $37,4 \%$ & $20,2 \%$ & $26,0 \%$ & $16,3 \%$ \\
\hline & hommes & $\%$ & $100,0 \%$ & $32,6 \%$ & $20,9 \%$ & $27,4 \%$ & $19,1 \%$ \\
\hline & femmes & $\%$ & $100,0 \%$ & $42,7 \%$ & $19,5 \%$ & $24,4 \%$ & $13,3 \%$ \\
\hline \multirow{3}{*}{$\begin{array}{l}\text { Artistes francophones au } \\
\text { Canada moins le Québec }\end{array}$} & Total & $\%$ & $100,0 \%$ & $40,4 \%$ & $16,2 \%$ & $26,7 \%$ & $17,4 \%$ \\
\hline & hommes & $\%$ & $100,0 \%$ & $35,7 \%$ & $16,8 \%$ & $27,1 \%$ & $21,9 \%$ \\
\hline & femmes & $\%$ & $100,0 \%$ & $44,8 \%$ & $14,9 \%$ & $26,2 \%$ & $13,1 \%$ \\
\hline
\end{tabular}




\begin{tabular}{|c|c|c|c|c|c|c|c|}
\hline Groupe & & $\begin{array}{l}\text { Tranche de } \\
\text { revenu }\end{array}$ & avec revenu & $\begin{array}{l}\text { moins que } \\
10000 \$\end{array}$ & $\begin{array}{c}10000 \\
\text { à } 19999 \$\end{array}$ & $\begin{array}{c}20000 \\
\text { à } 49999 \$\end{array}$ & $\begin{array}{c}50000 \$ \\
\text { et plus }\end{array}$ \\
\hline \multirow[t]{3}{*}{ Canadiens } & Total & nombre & 18201265 & 4524050 & 2862805 & 6476535 & 4337880 \\
\hline & hommes & nombre & 9480555 & 2029015 & 1232870 & 3239920 & 2978750 \\
\hline & femmes & nombre & 8720710 & 2495035 & 1629935 & 3236610 & 1359130 \\
\hline \multirow[t]{3}{*}{ Francophones au Canada } & Total & nombre & 4258720 & 1000833 & 722193 & 1655305 & 880393 \\
\hline & hommes & nombre & 2228575 & 452055 & 311133 & 861048 & 604330 \\
\hline & femmes & nombre & 2030143 & 548780 & 411055 & 794250 & 276058 \\
\hline \multirow{3}{*}{$\begin{array}{l}\text { Francophones au Canada } \\
\text { moins le Québec }\end{array}$} & Total & nombre & 603338 & 141115 & 90603 & 209890 & 161740 \\
\hline & hommes & nombre & 308465 & 61745 & 39458 & 103368 & 103898 \\
\hline & femmes & nombre & 294868 & 79370 & 51145 & 106515 & 57838 \\
\hline \multirow[t]{3}{*}{ Canadiens } & Total & $\%$ & $100,0 \%$ & $24,9 \%$ & $15,7 \%$ & $35,6 \%$ & $23,8 \%$ \\
\hline & hommes & $\%$ & $100,0 \%$ & $21,4 \%$ & $13,0 \%$ & $34,2 \%$ & $31,4 \%$ \\
\hline & femmes & $\%$ & $100,0 \%$ & $28,6 \%$ & $18,7 \%$ & $37,1 \%$ & $15,6 \%$ \\
\hline \multirow[t]{3}{*}{ Francophones au Canada } & Total & $\%$ & $100,0 \%$ & $23,5 \%$ & $17,0 \%$ & $38,9 \%$ & $20,7 \%$ \\
\hline & hommes & $\%$ & $100,0 \%$ & $20,3 \%$ & $14,0 \%$ & $38,6 \%$ & $27,1 \%$ \\
\hline & femmes & $\%$ & $100,0 \%$ & $27,0 \%$ & $20,2 \%$ & $39,1 \%$ & $13,6 \%$ \\
\hline \multirow{3}{*}{$\begin{array}{l}\text { Francophones au Canada } \\
\text { moins le Québec }\end{array}$} & Total & $\%$ & $100,0 \%$ & $23,4 \%$ & $15,0 \%$ & $34,8 \%$ & $26,8 \%$ \\
\hline & hommes & $\%$ & $100,0 \%$ & $20,0 \%$ & $12,8 \%$ & $33,5 \%$ & $33,7 \%$ \\
\hline & femmes & $\%$ & $100,0 \%$ & $26,9 \%$ & $17,3 \%$ & $36,1 \%$ & $19,6 \%$ \\
\hline
\end{tabular}

\begin{tabular}{|c|c|c|c|c|c|c|c|c|c|}
\hline & Sexe & Total & $\begin{array}{c}\text { Arts } \\
\text { médiatiques }\end{array}$ & $\begin{array}{c}\text { Arts } \\
\text { visuels }\end{array}$ & $\begin{array}{l}\text { Musique et } \\
\text { chanson } 1\end{array}$ & $\begin{array}{l}\text { Musique et } \\
\text { chanson } 2\end{array}$ & Danse & $\begin{array}{l}\text { Lettres et } \\
\text { édition }\end{array}$ & Thếtre \\
\hline \multirow[t]{3}{*}{ Artistes canadiens } & Total $(\mathrm{N})$ & 119045 & 22370 & 17115 & 2315 & 33630 & 7330 & 24545 & 11740 \\
\hline & hommes & 57990 & 14230 & 7475 & 1505 & 16125 & 1060 & 11240 & 6355 \\
\hline & femmes & 61060 & 8140 & 9635 & 815 & 17510 & 6270 & 13305 & 5385 \\
\hline \multirow{3}{*}{$\begin{array}{l}\text { Artistes francophones } \\
\text { au Canada }\end{array}$} & Total & 23655 & 6185 & 3260 & 350 & 5933 & 1235 & 4263 & 2430 \\
\hline & hommes & 12350 & 3863 & 1505 & 253 & 3465 & 245 & 1858 & 1163 \\
\hline & femmes & 11310 & 2315 & 1763 & 100 & 2470 & 988 & 2408 & 1268 \\
\hline \multirow{3}{*}{$\begin{array}{l}\text { Artistes Francophones } \\
\text { au Canada moins le } \\
\text { Québec }\end{array}$} & Total & 3125 & 560 & 503 & 48 & 800 & 205 & 668 & 343 \\
\hline & hommes & 1415 & 283 & 223 & 35 & 405 & 40 & 280 & 150 \\
\hline & femmes & 1715 & 278 & 280 & 15 & 405 & 160 & 393 & 185 \\
\hline \multirow[t]{3}{*}{ Artistes canadiens } & Total $(\%)$ & $100,0 \%$ & $100,0 \%$ & $100,0 \%$ & $100,0 \%$ & $100,0 \%$ & $100,0 \%$ & $100,0 \%$ & $100,0 \%$ \\
\hline & hommes & $48,7 \%$ & $63,6 \%$ & $43,7 \%$ & $65,0 \%$ & $47,9 \%$ & $14,5 \%$ & $45,8 \%$ & $54,1 \%$ \\
\hline & femmes & $51,3 \%$ & $36,4 \%$ & $56,3 \%$ & $35,2 \%$ & $52,0 \%$ & $85,5 \%$ & $54,2 \%$ & $45,9 \%$ \\
\hline \multirow{3}{*}{$\begin{array}{l}\text { Artistes francophones } \\
\text { au Canada }\end{array}$} & Total & $100,0 \%$ & $100,0 \%$ & $100,0 \%$ & $100,0 \%$ & $100,0 \%$ & $100,0 \%$ & $100,0 \%$ & $100,0 \%$ \\
\hline & hommes & $52,2 \%$ & $62,4 \%$ & $46,2 \%$ & $72,3 \%$ & $58,4 \%$ & $19,8 \%$ & $43,6 \%$ & $47,9 \%$ \\
\hline & femmes & $47,8 \%$ & $37,4 \%$ & $54,0 \%$ & $28,6 \%$ & $41,6 \%$ & $80,0 \%$ & $56,5 \%$ & $52,2 \%$ \\
\hline \multirow{3}{*}{$\begin{array}{l}\text { Artistes Francophones } \\
\text { au Canada moins le } \\
\text { Québec }\end{array}$} & Total & $100,0 \%$ & $100,0 \%$ & $100,0 \%$ & $100,0 \%$ & $100,0 \%$ & $100,0 \%$ & $100,0 \%$ & $100,0 \%$ \\
\hline & hommes & $45,3 \%$ & $50,5 \%$ & $44,3 \%$ & $72,9 \%$ & $50,6 \%$ & $19,5 \%$ & $41,9 \%$ & $43,7 \%$ \\
\hline & femmes & $54,9 \%$ & $49,6 \%$ & $55,7 \%$ & $31,2 \%$ & $50,6 \%$ & $78,0 \%$ & $58,8 \%$ & $54,0 \%$ \\
\hline
\end{tabular}




\begin{tabular}{|c|c|c|c|c|c|c|c|c|c|}
\hline \multirow[t]{2}{*}{ Groupe } & \multirow[t]{2}{*}{ Plus haut certificat, diplôme ou grade } & \multicolumn{2}{|c|}{$\begin{array}{l}\text { Population 15+ } \\
\text { (nombres) }\end{array}$} & \multicolumn{2}{|c|}{$\begin{array}{l}\text { Artistes professionnels } \\
\text { (nombres) }\end{array}$} & \multicolumn{2}{|c|}{$\begin{array}{l}\text { Population } 15 \text { ans } \\
\text { et plus }(\%)\end{array}$} & \multicolumn{2}{|c|}{$\begin{array}{c}\text { Artistes } \\
\text { professionnels }(\%)\end{array}$} \\
\hline & & hommes & femmes & hommes & femmes & hommes & femmes & hommes & femmes \\
\hline \multirow[t]{7}{*}{ Canada } & Total & 12470790 & 13193440 & 64885 & 68875 & $100,0 \%$ & $100,0 \%$ & $100,0 \%$ & $100,0 \%$ \\
\hline & Aucun certificat, diplôme ou grade & 3004625 & 3093700 & 5220 & 4595 & $24,1 \%$ & $23,4 \%$ & $8,0 \%$ & $6,7 \%$ \\
\hline & Diplôme d'études secondaires ou l'équivalent & 3032870 & 3520560 & 14765 & 11925 & $24,3 \%$ & $26,7 \%$ & $22,8 \%$ & $17,3 \%$ \\
\hline & \begin{tabular}{|l|} 
Certificat ou diplôme d'apprenti ou d'une école \\
de métiers
\end{tabular} & 1778080 & 1007340 & 2690 & 2275 & $14,3 \%$ & $7,6 \%$ & $4,1 \%$ & $3,3 \%$ \\
\hline & $\begin{array}{l}\text { Certificat ou diplôme d'un collège, d'un cégep } \\
\text { ou d'un autre établissement d'enseignement non } \\
\text { universitaire }\end{array}$ & 1906340 & 2528800 & 12915 & 12850 & $15,3 \%$ & $19,2 \%$ & $19,9 \%$ & $18,7 \%$ \\
\hline & $\begin{array}{l}\text { Certificat ou diplôme universitaire inférieur au } \\
\text { baccalauréat }\end{array}$ & 479095 & 657055 & 4225 & 4860 & $3,8 \%$ & $5,0 \%$ & $6,5 \%$ & $7,1 \%$ \\
\hline & Certificat, diplôme ou grade universitaire & 2269785 & 2385985 & 25055 & 32350 & $18,2 \%$ & $18,1 \%$ & $38,6 \%$ & $47,0 \%$ \\
\hline \multirow{7}{*}{$\begin{array}{l}\text { Francophones } \\
\text { au Canada }\end{array}$} & Total & 2994235 & 3182910 & 13683 & 12518 & $100,0 \%$ & $100,0 \%$ & $100,0 \%$ & $100,0 \%$ \\
\hline & Aucun certificat, diplôme ou grade & 773848 & 812748 & 813 & 698 & $25,8 \%$ & $25,5 \%$ & $5,9 \%$ & $5,6 \%$ \\
\hline & Diplôme d'études secondaires ou l'équivalent & 611540 & 749228 & 2638 & 1860 & $20,4 \%$ & $23,5 \%$ & $19,3 \%$ & $14,9 \%$ \\
\hline & \begin{tabular}{|l|} 
Certificat ou diplôme d'apprenti ou d'une école \\
de métiers
\end{tabular} & 586268 & 376958 & 750 & 655 & $19,6 \%$ & $11,8 \%$ & $5,5 \%$ & $5,2 \%$ \\
\hline & $\begin{array}{l}\text { Certificat ou diplôme d'un collège, d'un cégep } \\
\text { ou d'un autre établissement d'enseignement non } \\
\text { universitaire }\end{array}$ & 443865 & 566208 & 3268 & 2575 & $14,8 \%$ & $17,8 \%$ & $23,9 \%$ & $20,6 \%$ \\
\hline & $\begin{array}{l}\text { Certificat ou diplôme universitaire inférieur au } \\
\text { baccalauréat }\end{array}$ & 116783 & 174908 & 1155 & 1088 & $3,9 \%$ & $5,5 \%$ & $8,4 \%$ & $8,7 \%$ \\
\hline & Certificat, diplôme ou grade universitaire & 461928 & 502868 & 5063 & 5673 & $15,4 \%$ & $15,8 \%$ & $37,0 \%$ & $45,3 \%$ \\
\hline \multirow{7}{*}{$\begin{array}{c}\text { Francophones } \\
\text { au Canada } \\
\text { moins le } \\
\text { Québec }\end{array}$} & Total & 414130 & 458078 & 1628 & 1875 & $100,0 \%$ & $100,0 \%$ & $100,0 \%$ & $100,0 \%$ \\
\hline & Aucun certificat, diplôme ou grade & 116003 & 119235 & 115 & 120 & $28,0 \%$ & $26,0 \%$ & $7,1 \%$ & $6,4 \%$ \\
\hline & Diplôme d'études secondaires ou l'équivalent & 87283 & 110505 & 333 & 338 & $21,1 \%$ & $24,1 \%$ & $20,5 \%$ & $18,0 \%$ \\
\hline & $\begin{array}{l}\text { Certificat ou diplôme d'apprenti ou d'une école } \\
\text { de métiers }\end{array}$ & 61813 & 34783 & 65 & 90 & $14,9 \%$ & $7,6 \%$ & $4,0 \%$ & $4,8 \%$ \\
\hline & $\begin{array}{l}\text { Certificat ou diplôme d'un collège, d'un cégep } \\
\text { ou d'un autre établissement d'enseignement non } \\
\text { universitaire }\end{array}$ & 67130 & 87453 & 290 & 305 & $16,2 \%$ & $19,1 \%$ & $17,8 \%$ & $16,3 \%$ \\
\hline & $\begin{array}{l}\text { Certificat ou diplôme universitaire inférieur au } \\
\text { baccalauréat }\end{array}$ & 13168 & 21778 & 155 & 105 & $3,2 \%$ & $4,8 \%$ & $9,5 \%$ & $5,6 \%$ \\
\hline & Certificat, diplôme ou grade universitaire & 68733 & 84325 & 673 & 895 & $16,6 \%$ & $18,4 \%$ & $41,3 \%$ & $47,7 \%$ \\
\hline
\end{tabular}

